\title{
Surdose de venlafaxine traitée par assistance cardiorespiratoire extracorporelle
}

\author{
Laurel Murphy MD, Jack Rasmussen MD, Nancy G. Murphy MD CM
}

Citation : CMAJ 2021 February 1;193:E167-70. doi : 10.1503/cmaj.201318-f

Voir la version anglaise de l'article ici : www.cmaj.ca/lookup/doi/10.1503/cmaj.201318

$\mathbf{U}$ ne femme de 18 ans a été trouvée à la maison par sa mère à la suite d'une surdose polymédicamenteuse intentionnelle. On a estimé que l'ingestion avait eu lieu 2-5 heures plus tôt. La patiente avait reçu un diagnostic de trouble de la personnalité limite et avait des antécédents de tentatives de surdose n'ayant jamais nécessité d'hospitalisation. En vérifiant les flacons de médicaments et les ordonnances connues de la patiente, nous avons calculé qu'elle avait ingéré les médicaments suivants : 120 comprimés de venlafaxine XR $150 \mathrm{mg}$ (18000 mg), 30 comprimés de fluoxétine $10 \mathrm{mg}$ (300 mg) et 10 comprimés de diazépam $5 \mathrm{mg}(50 \mathrm{mg})$.

Pendant le transport de la patiente vers l'hôpital, les ambulanciers ont noté des mouvements s'apparentant à des convulsions qui ont pris fin sans traitement. Au service des urgences, la patiente était somnolente et répondait aux consignes de manière intermittente. Elle avait une température de $34,9^{\circ} \mathrm{C}$, un pouls de 109 battements par minute, une fréquence respiratoire de 24 respirations par minute, une pression artérielle de $108 / 72 \mathrm{~mm} \mathrm{Hg}$ et une saturation en oxygène de $96 \%$ à l'air ambiant. Ses pupilles étaient dilatées. Il n'y avait pas de clonus, de rigidité ou d'hyperréflexie. La glycémie était normale, et une analyse toxicologique sanguine a montré que la patiente n'avait pas consommé d'acide acétylsalicylique, d'acétaminophène ou d'alcool. Nous n'avons pas réalisé d'analyse urinaire.

L'électrocardiogramme (ECG) initial est présenté à la figure 1. Peu après son arrivée, la patiente a eu des convulsions qui ont été traitées par l'administration intraveineuse de lorazépam $1 \mathrm{mg}$. Nous avons communiqué avec le centre antipoison provincial pour obtenir des conseils sur la prise en charge et le monitorage de la patiente; la décontamination était contre-indiquée, les voies respiratoires de la patiente n'étant pas protégées. Dans les 6 heures qui ont suivi, la patiente a eu 3 autres crises convulsives et sa température a augmenté à $38,5^{\circ} \mathrm{C}$. En raison de l'accroissement de ses besoins en oxygène, nous avons décidé de l'intuber. Elle a par la suite reçu des perfusions de fentanyl, de midazolam et de noradrénaline.

Trois heures après l'intubation, la tachycardie de la patiente s'est aggravée, comme le montre l'ECG à la figure 2. La patiente a par la suite développé une tachycardie à complexes larges (figure 3), et sa

\section{POINTS CLÉS}

- Les surdoses de venlafaxine peuvent être mortelles.

- Les signes de toxicité comprennent les convulsions, l'allongement de l'intervalle QTc et l'élargissement du complexe QRS.

- L'ingestion de grandes quantités de venlafaxine peut provoquer une myocardiopathie grave, mais réversible, nécessitant une assistance cardiorespiratoire extracorporelle.

- Les intoxications graves devraient susciter une discussion avec le centre antipoison local quant aux options de décontamination gastro-intestinale et à la prise en charge.

pression artérielle par palpation est descendue à $66 \mathrm{~mm} \mathrm{Hg}$. La cardioversion n'a pas fonctionné. Peu après, la patiente a connu une dissociation électromécanique de 9 minutes, suivie d'une autre de 7 minutes. Une échographie mobile commandée par l'intensiviste de garde a révélé une dysfonction biventriculaire profonde avec une fraction d'éjection du ventricule gauche estimée à moins de $10 \%$ malgré l'administration d'une forte dose de noradrénaline et d'adrénaline au moment de l'examen.

La patiente a été transférée aux soins intensifs, où elle a eu besoin de fortes doses de vasopresseurs et d'agents inotropes. Elle avait un taux de lactate extrêmement élevé. Elle a développé une hypotension progressive, et une échocardiographie transœsophagienne a révélé une fraction d'éjection calculée de $6 \%$. Pour remédier à la situation, elle a été mise sous assistance cardiorespiratoire extracorporelle, qui a été combinée à un traitement continu de suppléance rénale en raison d'une insuffisance rénale aiguë. Elle présentait des marqueurs biochimiques d'insuffisance hépatique aiguë, ainsi qu'une hypoglycémie nécessitant une perfusion de glucose.

Dans la semaine qui a suivi, la fonction cardiaque de la patiente s'est graduellement améliorée. Une échocardiographie au jour 4 a montré que la fraction d'éjection était maintenant $25 \%-30 \%$; la fonction systolique du ventricule gauche était toujours très faible. Au jour 7 , la fonction cardiaque et la fraction d'éjection étaient revenues à la normale. Puis, au jour 8 , la patiente a été sevrée de l'assistance cardiorespiratoire 
extracorporelle et décanulée. Elle n'avait pas reçu de nourriture ou de médicaments par voie entérale et n'avait pas été à la selle depuis son admission. L'aspiration gastrique a fait ressortir de nombreux fragments de pilules ressemblant à des billes (figure 4). Un lavage gastrique retardé a été recommandé par le centre antipoison en raison de l'indisponibilité d'une endoscopie immédiate, et une irrigation intestinale totale n'a pas pu être effectuée, faute de péristaltisme. De nombreuses billes ont été aspirées. L'endoscopie supérieure réalisée le lendemain était normale, outre quelques billes résiduelles.

La patiente a été extubée au jour 13. Sa fonction rénale s'étant rétablie, l'hémodialyse a été arrêtée. Sa fonction neurologique était revenue à l'état préadmission. La patiente a été suivie par l'équipe psychiatrique à l'interne, et une thérapie comportementale dialectique a été commencée en consultation externe. Elle a reçu son congé de l'hôpital 43 jours après son admission.

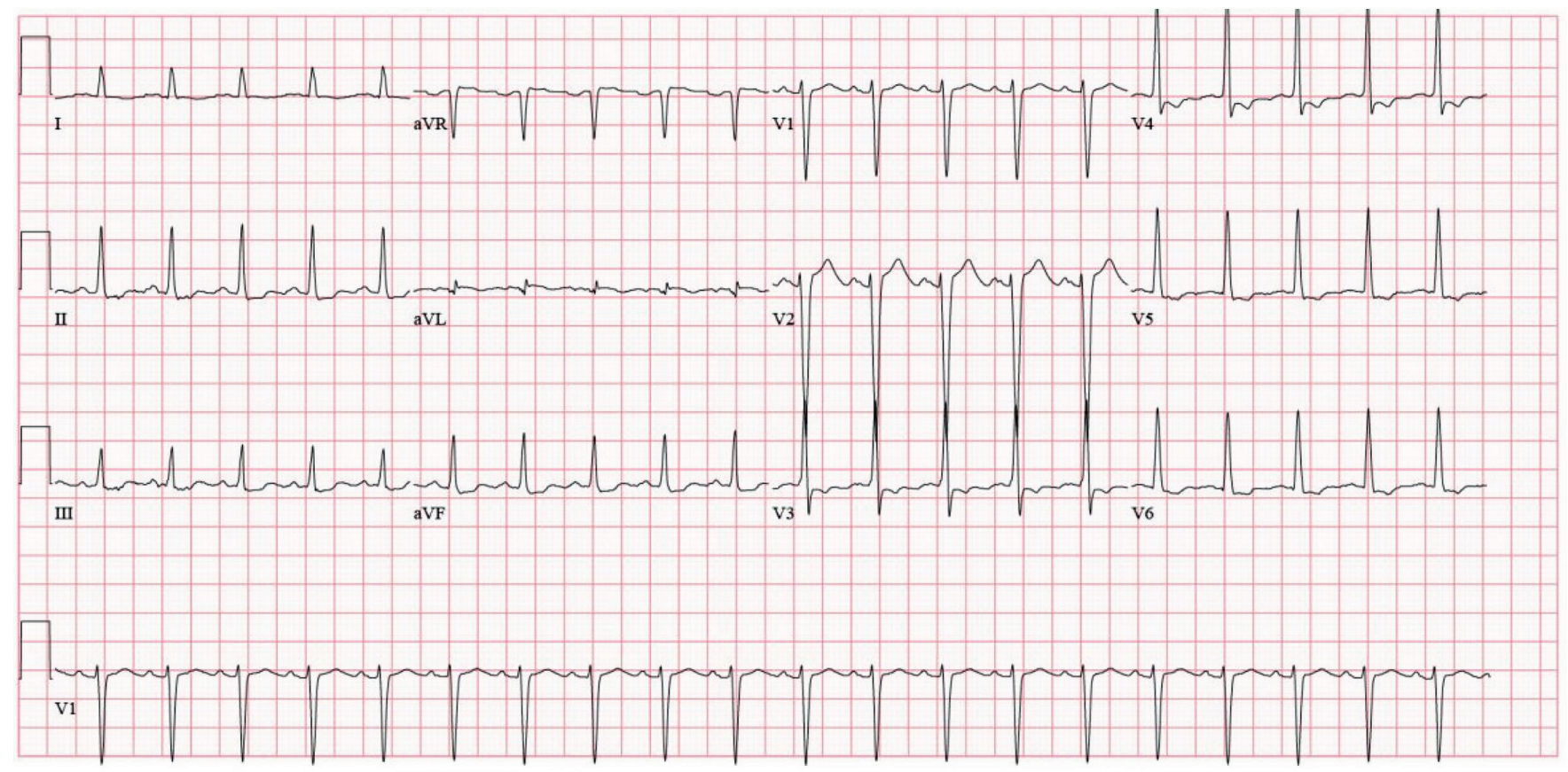

Figure 1 : Électrocardiogramme d'une femme de 18 ans à l'admission au service des urgences : intervalle QTc de 427 ms, intervalle QRS de 100 ms.

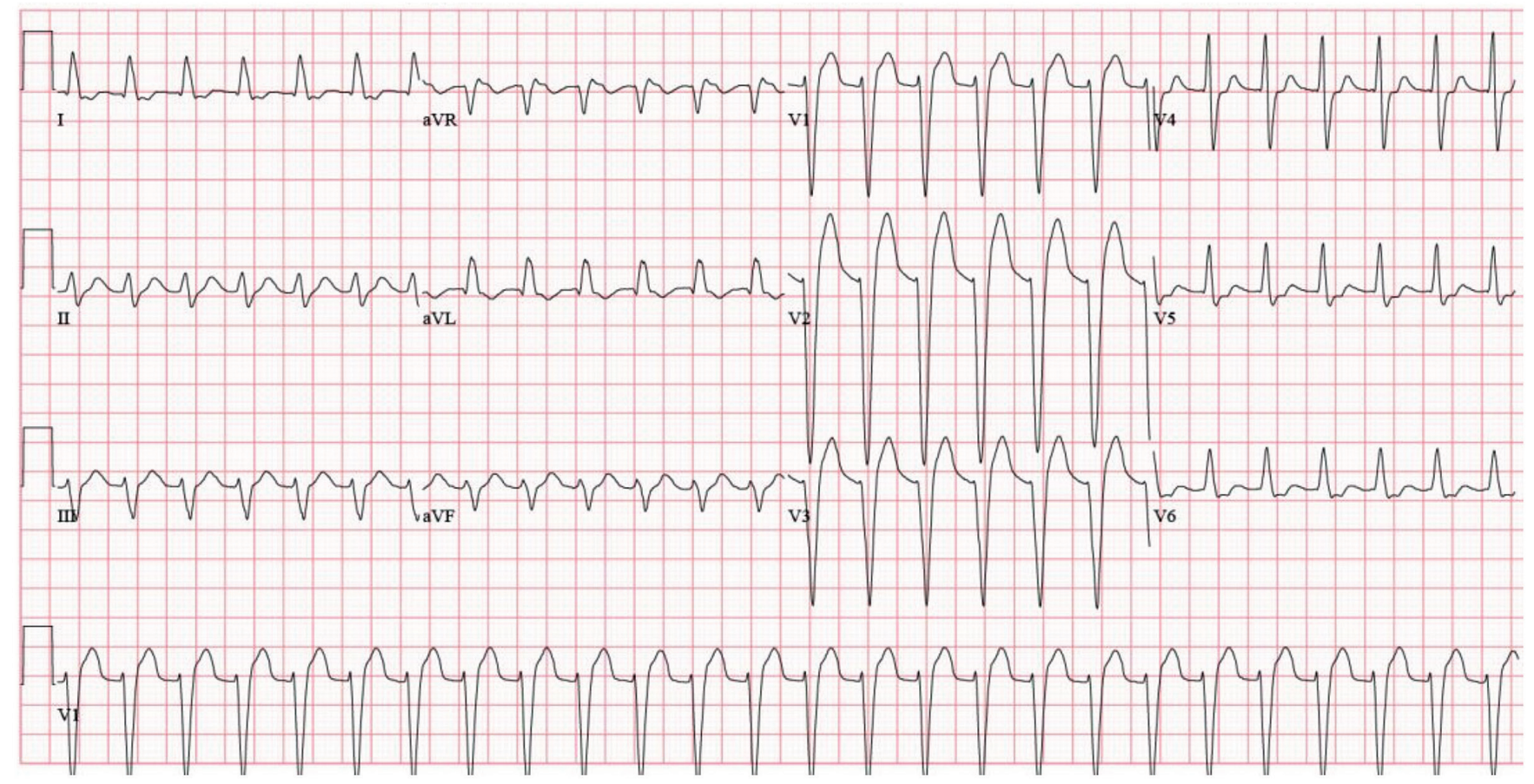

Figure 2 : Électrocardiogramme effectué environ 10 heures après l'arrivée de la patiente au service des urgences : intervalle de QTc de 531 ms, intervalle QRS de $160 \mathrm{~ms}$. 


\section{Discussion}

La venlafaxine est un inhibiteur de la recapture de la sérotonine/ noradrénaline couramment prescrite pour la dépression modérée à grave. Ce médicament est disponible en formule à libération immédiate ou prolongée. La formule à libération prolongée est offerte sous forme de comprimés ou de capsules de gélatine remplies de billes blanches ou blanchâtres. La venlafaxine XR est le seul élément ingéré qui contenait des billes dans le cas présenté ici. Il était assez probable que les billes recueillies dans le tractus gastro-intestinal soient venues de la venlafaxine $X R$, mais elles n'ont pas été analysées. La toxicité de la venlafaxine est plus faible que celle des antidépresseurs tricycliques, mais beaucoup plus élevée que celle des inhibiteurs sélectifs du recaptage de la sérotonine (ISRS) ${ }^{1}$. La venlafaxine est l'un des antidépresseurs non ISRS comportant le plus grand risque d'allongement de l'intervalle QT lors d'une surdose, accompagné d'un risque de décès cardiaque soudain causé par les torsades de pointes².

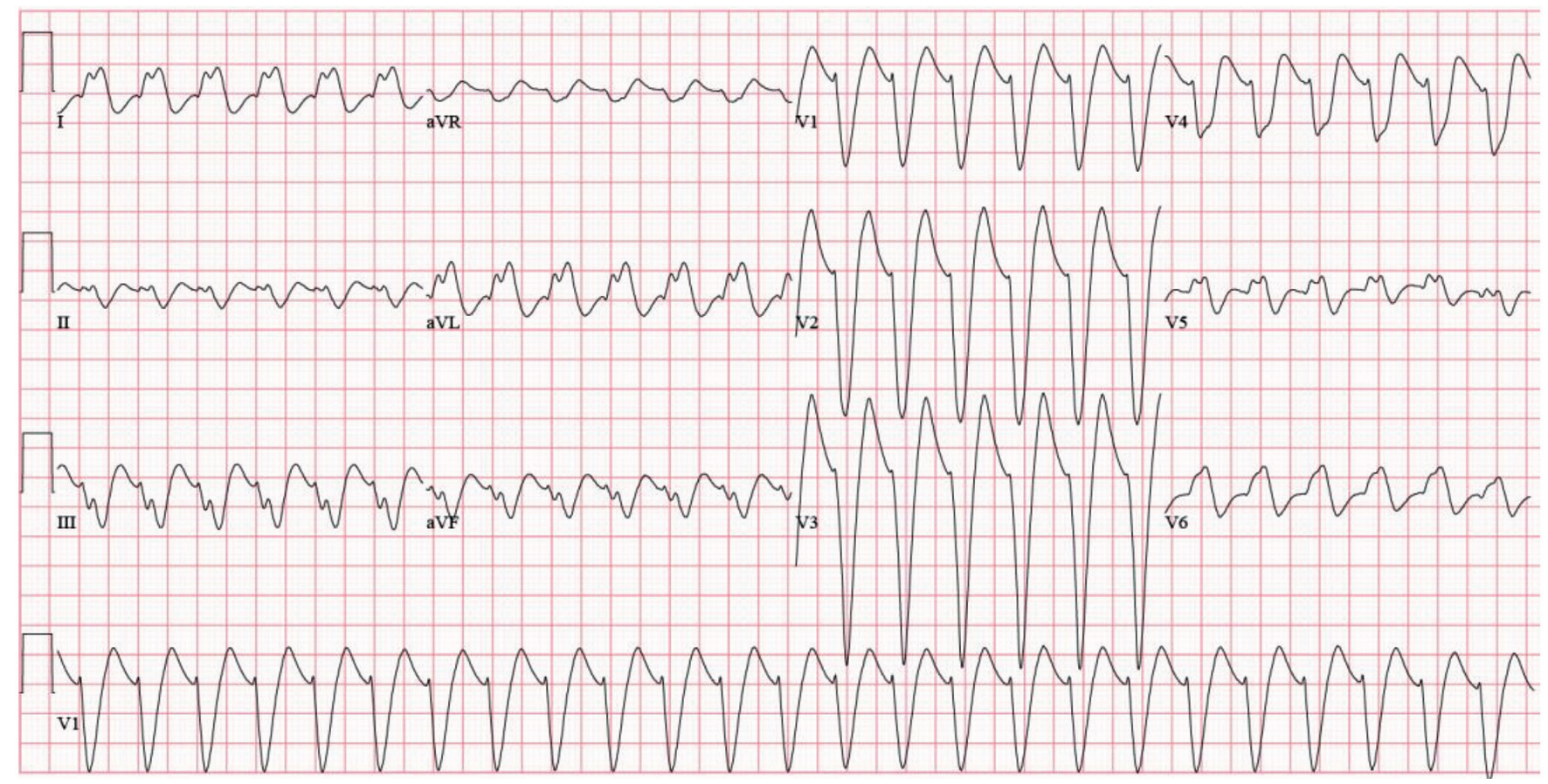

Figure 3 : Tachycardie à complexes larges chez la même patiente.

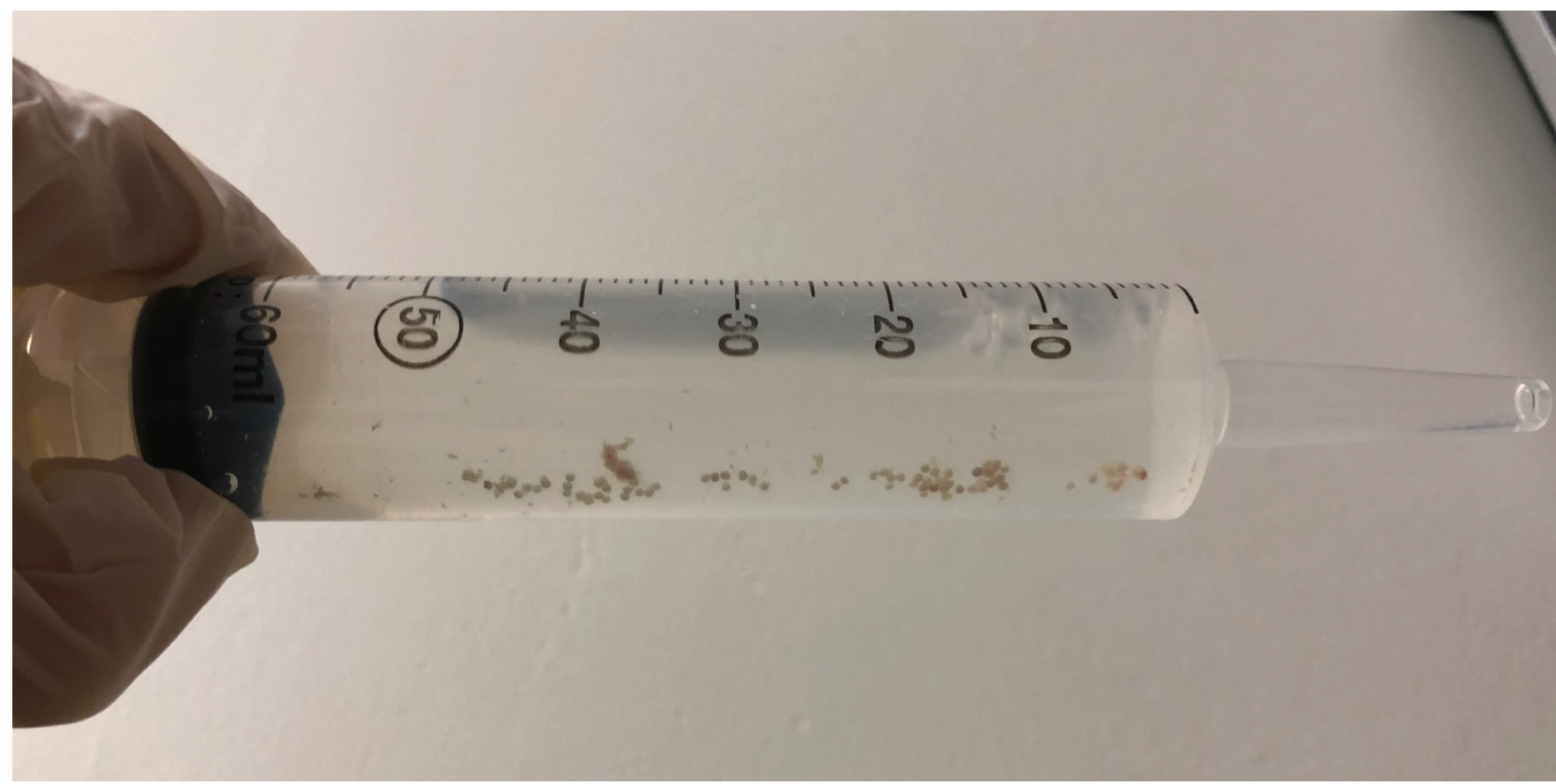

Figure 4 : Contenu du lavage gastrique réalisé au jour 8 . 


\section{Convulsions}

Les convulsions sont une séquelle courante de la surdose de venlafaxine, bien que quelques rares cas de convulsions associées à la venlafaxine à des doses thérapeutiques aient été signalés. Une revue rétrospective de 953 ingestions de venlafaxine réalisée par le California Poison Control System montre que des convulsions sont survenues dans $12,9 \%$ des cas $^{3}$. Les recommandations actuelles des centres antipoison, bien qu'elles varient, sont une mise en observation de 6 heures pour les formules à libération immédiate et 18-24 heures pour les formules à libération prolongée ${ }^{3}$.

\section{Toxicité cardiaque}

La surdose de venlafaxine est associée à la tachycardie, ainsi qu'à un allongement de l'intervalle QT et à un élargissement du complexe QRS proportionnels à la dose. Ces signes étaient tous présents chez la patiente. En cas d'ingestion majeure de plus de $8 \mathrm{~g}$, la toxicité cardiaque pourrait être plus prononcée ${ }^{4}$ (la patiente avait ingéré $18 \mathrm{~g}$ ). Le présent cas montre une progression claire des intervalles QTc et QRS. À l'admission, les 2 intervalles étaient normaux : l'intervalle QTc était de $427 \mathrm{~ms}$, et l'intervalle QRS, de $100 \mathrm{~ms}$ (figure 1). L'ECG réalisé 10 heures après l'arrivée de la patiente au service des urgences montre un allongement de l'intervalle QTc à $531 \mathrm{~ms}$ et un élargissement du complexe QRS à $160 \mathrm{~ms}$ (figure 2).

La surdose de venlafaxine a également été associée à de nombreux rapports de myocardiopathie réversible. Le cas présenté ici est un exemple extrême de cette situation : la patiente a eu besoin d'assistance cardiorespiratoire extracorporelle pendant 8 jours, le temps que sa fonction cardiaque s'améliore. À notre connaissance, 3 autres rapports dans la littérature scientifique traitent de l'utilisation réussie de l'assistance cardiorespiratoire extracorporelle pour une ingestion de venlafaxine d'ampleur semblable $(17,5 \mathrm{~g} \text { à } 20 \mathrm{~g})^{5}$. Le mécanisme de la toxicité cardiaque n'est pas entièrement compris, mais 2 théories ont été proposées. La première est que la venlafaxine inhibe les canaux calciques des myocytes ventriculaires de manière proportionnelle à la dose, ce qui provoque une sidération myocardique ${ }^{6}$. Les caractéristiques de cette inhibition des canaux calciques sont différentes de celles observées avec des antidépresseurs tricycliques ou des antiarythmisants de classe I. La deuxième théorie proposée pour expliquer la toxicité cardiaque est que l'excès de stimulation adrénergique produit une sidération myocardique semblable à la cardiomyopathie de tako-tsubo ${ }^{7}$.

\section{Décontamination gastro-intestinale}

La surdose d'un médicament oral à libération prolongée peut faire en sorte qu'une quantité importante de médicament non absorbé demeure dans le tractus gastro-intestinal pour une longue période. Bien que la décontamination gastro-intestinale soit controversée, elle a des bienfaits dans certaines situations ${ }^{8}$. Elle a pour principe fondamental de réduire ou de prévenir l'absorption du médicament, pour ainsi en limiter les effets toxiques potentiels. Comme il y a peu de données sur l'efficacité de cette intervention, il faut en peser les risques et les bienfaits potentiels dans tous les cas. En général, les contre-indications de la décontamination gastro-intestinale sous toutes ses formes comprennent les voies respiratoires non protégées, les vomissements répétés, un tractus gastro-intestinal non intact (iléus, obstruction), l'instabilité hémodynamique et un risque important d'aspiration (ingestion d'hydrocarbures) ${ }^{9}$.

Le lavage gastrique est une méthode de décontamination gastro-intestinale rarement utilisée qui a été largement remplacée par des interventions moins effractives, comme l'administration de charbon activé et l'irrigation intestinale totale. Dans les dernières années, le lavage endoscopique a été utilisé pour retirer des résidus de médicaments du tractus gastro-intestinal, mais il n'existe aucune recommandation claire quant à la sélection des patients pour cette procédure. Dans le cas qui nous intéresse, bien qu'on ait aspiré une grande quantité de résidus de médicaments 8 jours après l'ingestion, on ne peut dire s'il restait des médicaments actifs. Cependant, la seule présence de ces résidus indique que dans certains cas d'ingestion importante de médicaments à libération modifiée, l'absorption peut être retardée ou prolongée; il faudrait donc rapidement discuter de la possibilité de décontamination précoce avec le centre antipoison. Malheureusement, notre patiente avait des contre-indications à la décontamination à son admission (voies respiratoires non protégées) et après l'intubation (instabilité hémodynamique), ce qui souligne les limites de cette intervention.

\section{Conclusion}

La surdose de venlafaxine peut entraîner une toxicité mortelle, prenant le plus souvent la forme d'un collapsus cardiovasculaire. Les patients qui ingèrent une grande quantité de ce médicament peuvent développer une myocardiopathie grave, mais réversible, qui nécessite une assistance cardiorespiratoire extracorporelle. Nous suggérons donc que tout patient ayant ingéré une importante quantité de venlafaxine (> 8-10 g) subisse une échocardiographie tôt après l'admission, et que cet examen soit répété si l'état hémodynamique du patient empire. Étant donné le rôle salvateur joué par l'assistance cardiorespiratoire extracorporelle chez notre patiente, nous conseillons aussi de transférer les patients victimes d'une surdose majeure vers un centre où ce traitement peut être fourni au besoin. La consultation précoce avec un centre antipoison est aussi fortement recommandée pour discuter des options de décontamination gastro-intestinale et pour tirer parti de l'expertise de son personnel dans l'anticipation et la prise en charge d'une toxicité grave.

\section{Références}

1. Hawton K, Bergen H, Simkin S, et al. Toxicity of antidepressants: rates of suicide relative to prescribing and non-fatal overdose. $\mathrm{Br} J$ Psychiatry 2010;196:354-8.

2. Jasiak NM, Bostwick JR. Risk of QT/QTc prolongation among newer non-SSRI antidepressants. Ann Pharmacother 2014;48:1620-8.

3. Vo KT, Merriman AJ, Wang RC. Seizure in venlafaxine overdose: a 10-year retrospective review of the California poison control system. Clin Toxicol (Phila) 2020;58:984-90.

4. Isbister GK. Electrocardiogram changes and arrhythmias in venlafaxine overdose. Br J Clin Pharmacol 2009;67:572-6.

5. Marquetand C, Langer HF, Klein JP, et al. The use of extracorporeal life support in a patient suffering from venlafaxine intoxication: a case report. J Crit Care Med (Targu Mures) 2020;6:120-3. 
6. Khalifa M, Daleau P, Turgeon AJ. Mechanism of sodium channel block by venlafaxine in guinea pig ventricular myocytes. J Pharmacol Exp Ther 1999;291: 280-4.

7. Vasudev R, Rampal U, Patel H, et al. Selective serotonin-norepinephrine reuptake inhibitors-induced takotsubo cardiomyopathy. N Am J Med Sci 2016;8:312-5.

8. Livshits Z, Sampson BA, Howland MA, et al. Retained drugs in the gastrointestinal tracts of deceased victims of oral drug overdose. Clin Toxicol (Phila) 2015;53:113-8.

9. Benson BE, Hoppu K, Troutman WG, et al. Position paper update: gastric lavage for gastrointestinal decontamination. Clin Toxicol (Phila) 2013;51:140-6.

La section Études de cas présente de brefs rapports de cas à partir desquels des leçons claires et pratiques peuvent être tirées. Les rapports portant sur des cas typiques de problèmes importants, mais rares ou sur des cas atypiques importants de problèmes courants sont privilégiés. Chaque article commence par la présentation du cas (500 mots maximum), laquelle est suivie d'une discussion sur l'affection sous-jacente (1000 mots maximum). La soumission d'éléments visuels (p. ex., tableaux des diagnostics différentiels, des caractéristiques cliniques ou de la méthode diagnostique) est encouragée. Le consentement des patients doit impérativement être obtenu pour la publication de leur cas. Renseignements destinés aux auteurs : www.cmaj.ca.

\section{Intérêts concurrents : Aucun déclaré.}

Cet article a été révisé par des pairs.

Les auteurs ont obtenu le consentement de la patiente.

Affiliations : Départements de médecine d'urgence et de soins intensifs (L. Murphy) et de chirurgie; Division de chirurgie plastique, Département de soins intensifs (Rasmussen); Département de médecine d'urgence et IWK Regional Poison Centre (N. Murphy), Université Dalhousie, Halifax, N-É.

Collaborateurs : Tous les auteurs ont contribué à l'élaboration et à la conception de l'étude, ont rédigé le manuscrit et en ont révisé de façon critique le contenu intellectuel important; ils ont donné leur approbation finale pour la version destinée à être publiée et assument l'entière responsabilité de tous les aspects du travail.

Propriété intellectuelle du contenu : Il s'agit d'un article en libre accès distribué conformément aux modalités de la licence Creative Commons Attribution (CC BY-NC-ND 4.0), qui permet l'utilisation, la diffusion et la reproduction dans tout médium à la condition que la publication originale soit adéquatement citée, que l'utilisation se fasse à des fins non commerciales (c.-à-d., recherche ou éducation) et qu'aucune modification ni adaptation n'y soit apportée. Voir: https://creativecommons.org/licenses/by-nc-nd/4.0/deed.fr.

Correspondance : Laurel Murphy, laurel.murphy@dal.ca 